\title{
Corrigendum
}

\section{Corrigendum to "Preparation of Associative Polyurethane Thickener and Its Thickening Mechanism Research"}

\author{
Gao Nan, ${ }^{1,2}$ Zhang Zhuo, $^{2}$ and Dong Qingzhi ${ }^{1}$ \\ ${ }^{1}$ East China University of Science and Technology, Shanghai 200237, China \\ ${ }^{2}$ Shanghai Institute of Technology, Shanghai 200235, China \\ Correspondence should be addressed to Gao Nan; gaonanxf@sit.edu.cn and Dong Qingzhi; qzhdong@ecust.edu.cn \\ Received 7 November 2015; Accepted 30 November 2015 \\ Copyright (C) 2015 Gao Nan et al. This is an open access article distributed under the Creative Commons Attribution License, which \\ permits unrestricted use, distribution, and reproduction in any medium, provided the original work is properly cited.
}

In the research article titled "Preparation of Associative Polyurethane Thickener and Its Thickening Mechanism Research" [1], the correct correspondence authors shall be shown as above.

The correspondence authors have lost one name (Dong Qingzhi); the correct correspondence authors have been shown in the front matter of the paper.

\section{References}

[1] G. Nan, Z. Zhuo, and D. Qingzhi, "Preparation of associative polyurethane thickener and its thickening mechanism research," Journal of Nanomaterials, vol. 2015, Article ID 137646, 7 pages, 2015. 

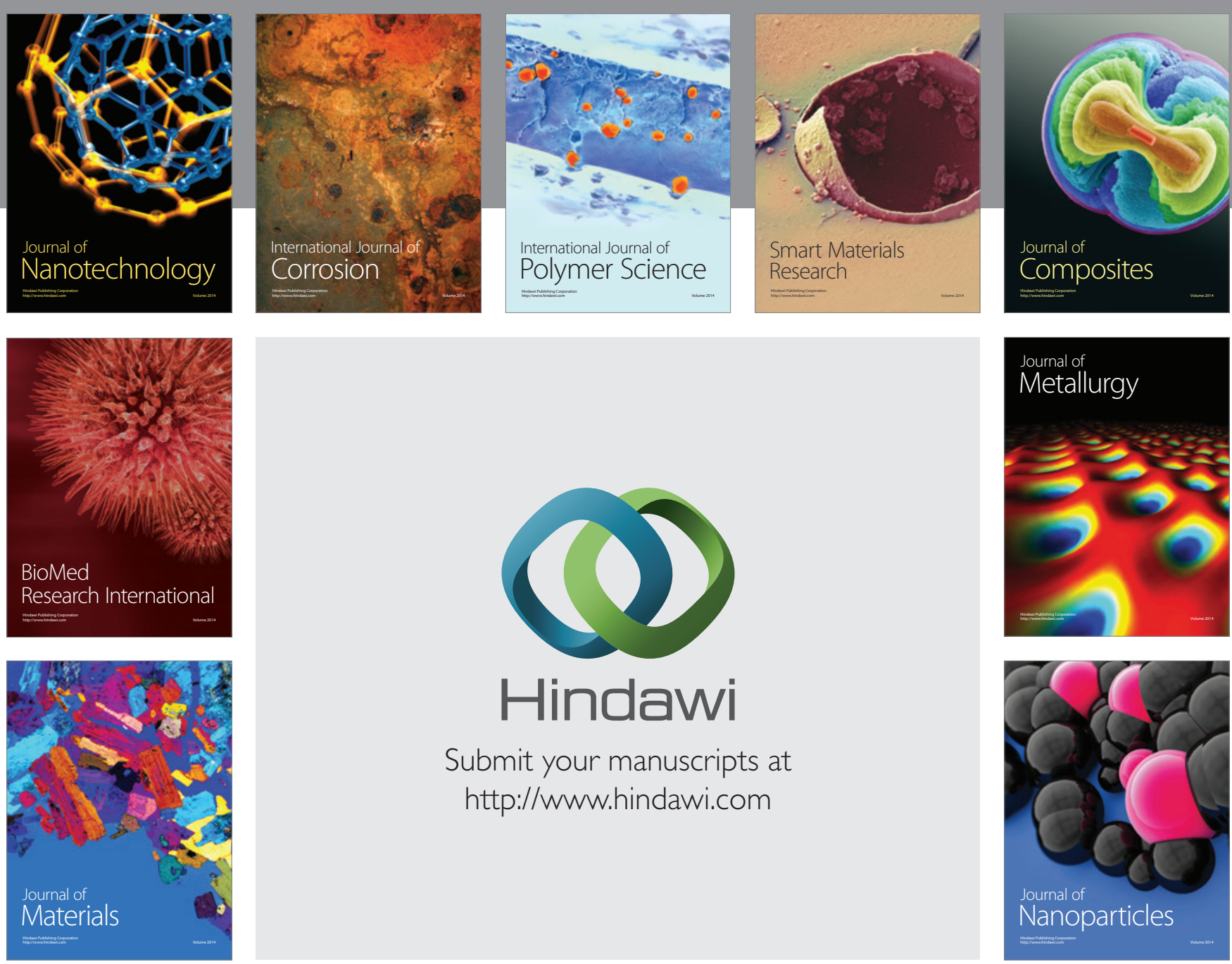

Submit your manuscripts at http://www.hindawi.com
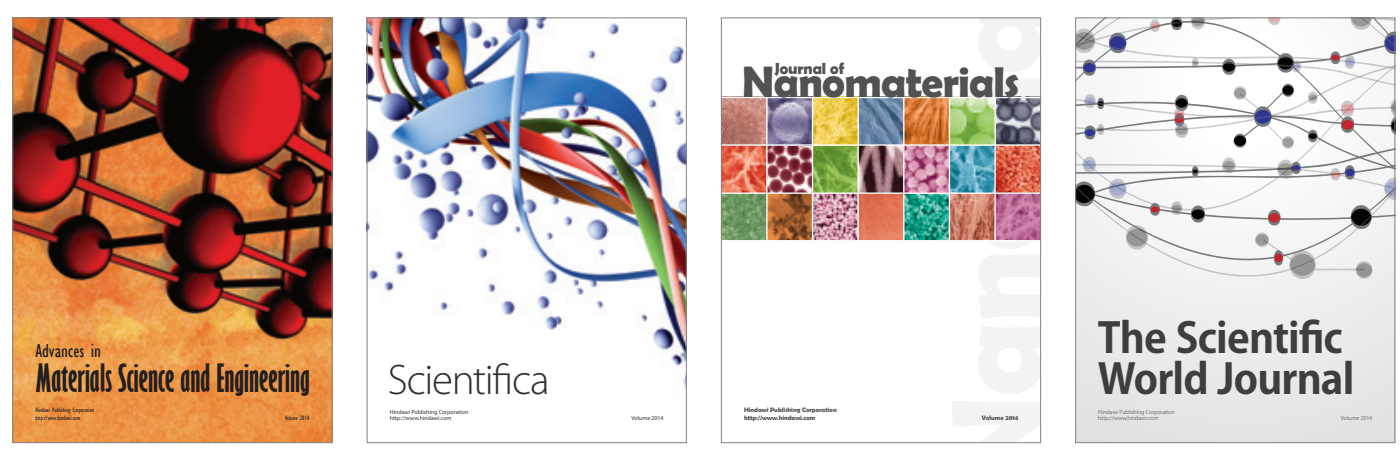

\section{The Scientific World Journal}
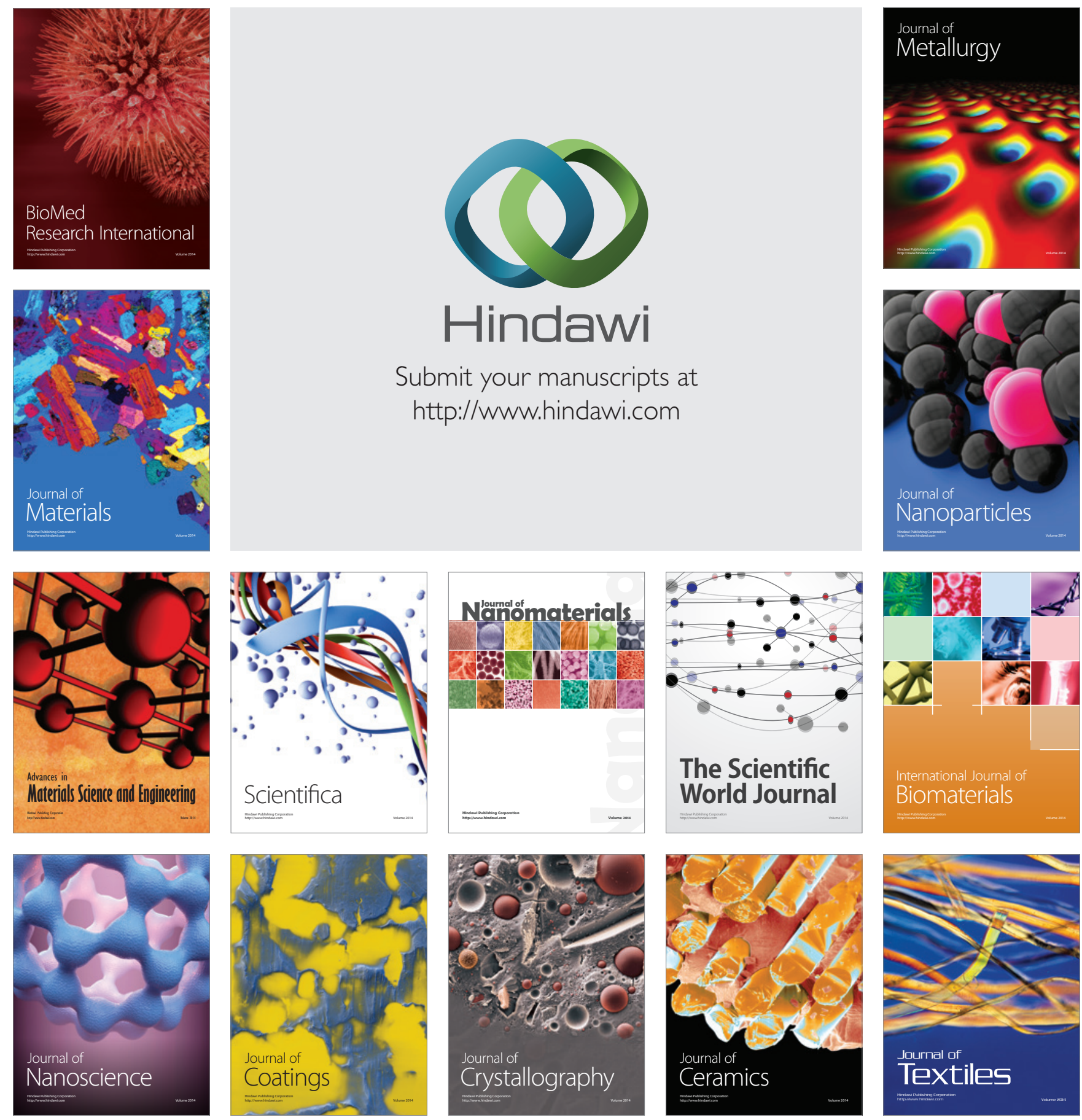